\title{
THE HYODO-KATO THEOREM FOR RATIONAL HOMOTOPY TYPES
}

\author{
Minhyong Kim AND RichaRd M. Hain
}

\begin{abstract}
The Hyodo-Kato theorem relates the De Rham cohomology of a variety over a local field with semi-stable reduction to the log crystalline cohomology of the special fiber. In this paper we prove an analogue for rational homotopy types. In particular, this gives a comparison isomorphism for fundamental groups.
\end{abstract}

\section{Introduction}

Let $A$ be a complete discrete valuation ring with perfect residue field $k$ of characteristic $p>0$ and fraction field $F$. Denote by $W$ the ring of Witt vectors of $k$ and by $K$ the fraction field of $W$. Endow $A$ with the $\log$ structure $A-0 \hookrightarrow A$ and let $X$ be a proper smooth connected fine saturated log scheme over $A$ with generic fiber $X_{F}$ and special fiber $Y$ which we assume to be of Cartier type ([6] 2.12). The Hyodo-Kato theorem ([6] Theorem 5.1) says

$$
H_{D R}^{i}\left(X_{F}\right) \simeq H_{c r}^{i}(Y / W) \otimes_{W} F
$$

where the cohomology groups appearing in the statement are algebraic De Rham cohomology on the left and the crystalline cohomology of $Y$ with respect to $W$ (with the 'hollow' log structure) on the right.

We will prove a version of this theorem in the context of the unipotent rational homotopy types defined in [9]. The definitions will be reviewed in the next section, but let us state the main result here. If $A_{c r}(Y)$ denotes the crystalline rational homotopy type of $Y$ and $A_{D R}\left(X_{F}\right)$ the De Rham rational homotopy type of $X_{F}$, then

\section{Theorem 1.1.}

$$
A_{D R}\left(X_{F}\right) \simeq A_{c r}(Y) \otimes F
$$

in the homotopy category of commutative differential graded algebras (CDGA's) over F.

In [9], we proved this result essentially when $A$ has the trivial log structure. Let $x$ be a point of $X, x_{F}$ the correponding point on the generic fiber, and $y$ the reduction of $x$ to the special fiber. It is simple to check that the augmentations induced by $x_{F}$ and $y$ on the homotopy types are compatible. Hence, we get

Received April 14, 2004. 


\section{Corollary 1.2.}

$$
\pi_{1}^{d r}\left(X_{F}, x_{F}\right) \simeq \pi^{c r}(Y, y) \otimes_{K} F
$$

as pro-unipotent algebraic groups over $F$.

We also get results of the Artin-Mazur type [1] on higher homotopy groups of simply connected varieties. For this, let $X_{E}(1)$ and $X_{E}(2)$ be proper smooth geometrically-connected varieties over a number field $E$ equipped with normal crossing divisors $D_{E}(1)$ and $D_{E}(2)$. Denote by $\mathcal{O}_{E, v}$ the localization at a prime $v$ of the ring of integers $\mathcal{O}_{E}$ of $E$. Assume that $X_{E}(i)$ extends to a proper flat scheme $X(i)$ over $\mathcal{O}_{E, v}$ with special fibers $Y(i)$ and that $D_{E}(i)$ extends to a divisor $D(i)$ on $X(i)$ that is relatively of normal crossing. Further assume that $D(i)+Y(i)$ is a strict normal crossing divisor. View $X(i)$ as a log scheme with the $\log$ structures given by $D(i)+Y(i)$. Then $Y(i)$ also has the induced log structure.

Corollary 1.3. Suppose $Y(1) \simeq Y(2)$ as log schemes. Then for every embedding $\sigma: E \hookrightarrow \mathbf{C}$ such that the $\left(X_{E}(i)-D_{E}(i)\right) \otimes_{E} \mathbf{C}$ are simply-connected, their higher rational homotopy groups are isomorphic.

The hypothesis of the corollary is satisfied for example if

$$
X(1) \otimes \mathcal{O}_{E, v} / m_{v}^{2} \simeq X(2) \otimes \mathcal{O}_{E, v} / m_{v}^{2}
$$

by a map that preserves the divisors $D(i)+Y(i)$.

The proof of the theorem consists of choosing explicit CDGA representatives for the homotopy types and a few other intermediate complexes using embedding systems for crystalline cohomology, constructing explicit multiplicative maps between them and, thereby, 'exorcising the derived category' from the proof of the usual Hyodo-Kato isomorphism.

It is perhaps useful to think about the theorem in the following general context: Let $\mathcal{A}$ be the homotopy category of non-negatively graded CDGA's over some field and consider the forgetful functor

$$
R: \mathcal{A} \rightarrow \mathcal{C}
$$

to the derived category of complexes. Following the ideas of rational homotopy theory, one should think of objects $A$ in $\mathcal{A}$ as being rationally nilpotent topological spaces and $R(A)$ as being like the homotopy groups of the space. Given two objects $A, B$ and an isomorphism $g: R(A) \simeq R(B)$, we can then ask if there is in fact an isomorphism $f: A \rightarrow B$ such that $g=R(f)$. That is, we wish to prove a Whitehead-type theorem showing that an isomorphism of homotopy groups is induced by an actual isomorphism of spaces. In our situation, the usual Hyodo-Kato isomorphism says that $R\left(A_{D R}\left(X^{*}\right)\right) \simeq R\left(A_{c r}(Y) \otimes_{K} F\right)$ and our theorem says that this map can be lifted to a map of 'spaces.'

In the process of making maps explicit, one encounters the problem of assembling a collection of maps in (an increasing sequence of) finite characteristics into a single map of algebras. This is because the Hyodo-Kato isomorphism depends on the fact that repeated Frobenius twists of complexes computing crystalline 
cohomology with respect to two different log structures become increasingly close, i.e., isomorphic modulo increasingly high powers of $p$. That is, an inverse system of complexes related by the relative Frobenius and reduction mediates the Hyodo-Kato isomorphism. To deal with this difficulty we use a differential graded algebra version of homotopy direct limits, namely, the mapping telescope of an inverse system of maps. In fact, it will be necessary to, employ the notion of a 'twisted' inverse limit introduced by Ogus to define 'twisted' mapping telescopes and the key definition of an 'infinitely twisted' mapping telescope (obtained by taking a direct limit of twisted mapping telescopes). Once one has this machinery in place, the isogenies that occur in the usual proof fall into place nicely as isomorphisms of $\infty$-twisted mapping telescopes and the rest of the argument becomes quite short.

We remark that the proof of theorem 1.1 appears to clarify the cohomological Hyodo-Kato isomorphism as well: The rational homotopy types considered as complexes compute the usual cohomology groups (crystalline and De Rham). However, the proof given here, while just a modification of those of Hyodo-Kato and Ogus, possesses an advantage over them in that the maps involved are made completely explicit.

One issue we do not deal with in this paper is the dependence of the isomorphism stated in the theorem on the choice of a uniformizer for $A$. Although it is more or less clear that one gets the same kind of dependence as in the cohomological theorem, we prefer to discuss this in a subsequent paper together with a more detailed study of the monodromy operator and period isomorphisms.

A few words about our convention: For the most part we leave the log structure implicit and do not introduce separate notation to indicate their presence. The important exception of course is in the proof of theorem 1.1. A multiplicative quasi-isomorphism of CDGA's is just a quasi-isomorphism at the level of complexes that respects the multiplicative structure. A quasi-equivalence, on the other hand, is an isomorphism in the homotopy category of CDGA's.

\section{Brief review}

For precise definitions, we refer the reader to [9] sections 3 and 4, and the references therein. In this section, we will just recall at the superficial level some basic notation, the objects that we will need, and their main properties.

For concreteness, we concentrate on the situation described in the introduction. Therefore, $k$ is endowed with the log structure of the punctured point which is associated to the pre-log structure $\mathbf{N} \rightarrow k$ that sends 1 to 0 . We denote by $Y$ a smooth proper fine saturated log scheme of Cartier type over $k$ and $y: \operatorname{Spec}(k) \rightarrow Y$ a point of $Y$. (We remind the reader that this means in particular that it is a map of log schemes.)

The unipotent crystalline rational homotopy type $A_{c r}(Y)$ of $Y$ is defined by the following formula:

$$
A_{c r}(Y)=T W\left(W \omega_{Y}\right):=s_{T W}\left(\left(\varliminf_{\longleftarrow} \Gamma\left(G\left(W \omega_{Y}\right)\right)\right) \otimes_{W} K\right)
$$


The notation is that given a pro-sheaf $L, G(L)$ is a cosimplicial Godement resolution [3] for the étale topology, lim goes from the category of inverse systems $\left(M_{n}\right)_{n \in \mathbf{N}}$, where $M_{n}$ is a (cosimplicial) $W_{n}$-algebra, to the category of complete cosimplicial $W$-algebras, and finally, $s_{T W}$ is Navarro-Aznar's 'simple Thom-Whitney algebra' functor [11]. $W \omega_{Y}$ is the pro-sheaf of CDGA's consisting of the De Rham-Witt differential forms of $Y$ [6]. It is probably best at this point not to worry about the precise construction and just remember that

$$
T W(\cdot)=s_{T W}\left(\left(\lim _{\longleftarrow} \Gamma(G(\cdot)) \otimes\right)_{W} K\right)
$$

is a functor from the isogeny category of pro-sheaves of CDGA's over $W$ (that is, systems $\left(L_{n}\right)$ where a given level $L_{n}$ is a sheaf of CDGA's over $\left.W_{n}\right)$ to the homotopy category of CDGA's over $K$. As motivation for the language of homotopy types, we refer the reader to [10].

A basic property of the functor $s_{T W}$ is that for any cosimplicial CDGA $C$, $s_{T W}(C) \simeq s(C)$ in the derived category of complexes, where $s(\cdot)$ denotes the usual 'associated simple object' functor. Ostensibly, the definition depends on the choice of a surjective system of geometric points on $Y$. One can remove this dependence as follows. Denote for a moment $A_{S}$ the rational homotopy type constructed from a specific system $S$ of geometric points. Given two different systems $S_{1}$ and $S_{2}$, we can find a map $f: S_{1} \rightarrow S_{2}$ which certainly induces a multiplicative quasi-isomorphism

$$
f^{*}: A_{S_{2}} \simeq A_{S_{1}}
$$

To see that this map is independent of the choice of $f$, Consider $S_{3}:=S_{1} \times_{Y} S_{2}$ and the graph of $f, g_{f}: S_{1} \rightarrow S_{3}$. Denote by $\pi_{i}: S_{3} \rightarrow S_{i}$ the projection maps. Then $\pi_{i}^{*}: A_{S_{i}} \rightarrow A_{S_{3}}$ is a multiplicative quasi-isomorphism for each $i$ as is $g_{f}^{*}$ : $A_{S_{3}} \rightarrow A_{S_{1}}$. Then since $g_{f}^{*} \circ \pi_{1}^{*} ; A_{S_{1}} \rightarrow A_{S_{1}}$ is the identity, we get that $g_{f}^{*}=\left(\pi_{1}^{*}\right)^{-1}$ in the homotopy category. Therefore, $f^{*}=g_{f}^{*} \circ \pi_{2}^{*}=\left(\pi_{1}^{*}\right)^{-1} \circ \pi_{2}^{*}$ is independent of $f$ in the homotopy category. That is to say $A_{1}$ and $A_{2}$ are canonically quasiequivalent. This justifies omitting the system of points from the notation for the homotopy type. We will omit similar obvious arguments in a few other places of the papers. We remark that this proof was suggested by the referee, correcting an earlier error.

The (unipotent) De Rham rational homotopy type of $X_{F}$ is defined by

$$
A_{D R}(X)=T W\left(\Omega_{X_{F} / F}\right):=s_{T W} \Gamma\left(G\left(\Omega_{X_{F} / F}\right)\right)
$$

where the Godement resolution is now taken on $X_{F}$. But Grothendieck's existence theorem $([4], 5.1 .2)$ implies that

$$
A_{D R}(X) \simeq s_{T W}\left(\left(\lim _{\longleftarrow} \Gamma\left(G\left(\Omega_{\hat{X} / A}\right)\right)\right) \otimes_{A} F\right)
$$

where the formal completion $\Omega_{\hat{X} / A}$ of the De Rham complex of $X / A$ is regarded as a pro-sheaf on $Y$. It is the latter object that we will compare to $A_{c r}$.

One can compute $A_{c r}$ using the 'crystalline complex' associated to an embedding system ([6] 2.18): Let $(Y, Z$.) be a pair of simplicial schemes that fit into a 
diagram

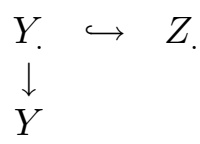

where $Y$. is a simplicial hypercovering that satisfies cohomological descent for the étale topology which we equip with the log structure pulled back from that of $Y, Y \hookrightarrow Z$. is a closed embedding of formal $\log$ schemes, and $Z$. is smooth formal $\log$ scheme over $W$. Here, $W$ is equipped with the 'hollow' [12] log structure associated to the monoid $\mathbf{N}$ and the zero map $\mathbf{N} \rightarrow W$. Then the associated crystalline complex is by definition

$$
C\left(Y, Z_{.}\right):=\Omega_{Z . / W} \otimes_{\mathcal{O}_{Z} .} D_{Y .}(Z .)
$$

Here, $D_{Y}(Z$. $)$ is the divided power envelop of $Y$. in $Z$. Regard $C(Y, Z$.) as a simplicial pro-CDGA on $Y$. Then we also defined $T W(C(Y, Z)$.$) in this setting$ and we have a quasi-equivalence (i.e., an isomorphism in the homotopy category):

$$
T W\left(C\left(Y_{.}, Z .\right)\right) \simeq T W\left(W \omega_{Y}\right)
$$

The functor $T W$ (Thom-Whitney) can be defined in a more general setting, as we have already done in the definition of $A_{D R}$ for example, but also for crystalline complexes over more general bases. So if $Y / S$ is a fine saturated smooth log scheme over an affine base $S=\operatorname{Spec}(R)$ and $S \hookrightarrow T=\operatorname{Spec}(B)$ is an exact immersion of formal $\log$ schemes where $B$ is flat over $\mathbf{Z}$, then to any embedding system $(Y ., Z$.) as above with $Z$. smooth over $B$, we can associate the crystalline complex $C(Y, Z$. $)$ and the Thom-Whitney algebra $T W(C(Y, Z)$.$) which ends up$ as a CDGA over $B \otimes \mathbf{Q}$. This algebra is in fact canonically independent of the embedding system: Any two embedding systems can be dominated by a third giving rise to a quasi-equivalence which, in turn, is independent in the homotopy category of the dominating system, as in our earlier argument.

\section{Mapping telescopes}

Now we will go on to define the (twisted) mapping telescopes of inverse systems of complete $W$-algebras.

First, if $f: A \rightarrow B$ is a map of (complete) CDGA's over $W$, we define the mapping cylinder that fits into a diagram

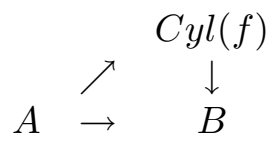

as follows: Denote by $I$ the formal divided power De Rham complex of $W<<$ $x>>$, the p-adic completion of the divided power polynomial algebra over $\mathrm{W}$, which therefore is concentrated in degrees 0 and 1 . Consider the two augmentation maps $e_{0}, e_{p}: I \rightarrow \mathbf{Z}$ that evaluate functions in $I^{0}$ at 0 and $p$, respectively, and sends $I^{1}$ to zero. Hence $B \otimes I$ is likewise equipped with two maps to $B$ which we also denote by $e_{0}$ and $e_{p}$. Here and henceforward, all tensor products are topological. The mapping cylinder $C y l(f)$ of $f$ is defined to be the subalgebra 
of $A \oplus B \otimes I$ consisting of elements $(a, b)$ such that $f(a)=e_{0}(b)$. Notice that the map $C y l(f) \rightarrow B$ induced by $e_{p}: B \otimes I \rightarrow B$ becomes surjective after tensoring with Q: for any $b \in B,(0, x b / p)$ is in $C y l(f) \otimes \mathbf{Q}$ and maps to $b$. On the other hand, integrally, we can only say that if $b \in B$, then $p b$ is in the image of $e_{p}$. The map $A \rightarrow C y l(f)$ given by $a \mapsto(a, f(a))$ induces a q.i. In fact, the projection $C y l(f) \rightarrow A$ to the first component is a chain homotopy inverse. Now, given an inverse system of maps

$$
\text { A. }: \cdots \rightarrow A_{3} \rightarrow A_{2} \rightarrow A_{1}
$$

indexed by the positive integers, it is clear how to construct the mapping telescope $\operatorname{Tel}\left(A_{\text {. }}\right)$. It is the inverse limit of the inverse system defined inductively by putting $T e l_{1}=A_{1}$ and

$$
T e l_{i+1}:=\operatorname{Cyl}\left(A_{i+1} \rightarrow T e l_{i}\right)
$$

where the map $A_{i+1} \rightarrow T e l_{i}$ is the composite $A_{i+1} \rightarrow A_{i} \rightarrow T e l_{i}$. The construction of $\mathrm{Tel}$ is clearly functorial for inverse systems of CDGA's. In particular, one can apply the construction to inverse systems of cosimplicial CGDA's or bicosimplicial CDGA's. Also, there is a map of inverse systems $A \rightarrow T e l$. giving rise to a functorial map $\lim A . \rightarrow T e l$.

We will also need the 'twisted' inverse limit construction of Ogus ([12] p.203): Given an inverse system $L$. of $W$-modules indexed by $\mathbf{N}$ and an integer $m$, let $\varliminf^{m} L$. be the collection of elements $\left(a_{i}\right), a_{i} \in L_{i}$, such that $a_{i} \mapsto p^{m} a_{i-1}$. If $n \geq$ $m$, then we have natural maps $\varliminf^{m} L . \longleftarrow^{n}{ }^{n} L$. given by $\left(a_{i}\right) \mapsto\left(p^{(n-m) i} a_{i}\right)$. That is, the twisted inverse limits form a directed system.

Using these, we can also construct the $m$-twisted telescope

$$
\operatorname{Tel}^{m}(A .)=\varliminf^{m} \operatorname{Tel}_{i}(A .)
$$

as well as the infinitely twisted telescope

$$
T e l^{\infty}(A .)=\varliminf_{m} T e l^{m}(A .)
$$

Clearly, we have functorial maps $\varliminf^{m} A . \rightarrow \operatorname{Tel}^{m}(A$. $)$ and

$$
\varliminf_{m} \varliminf_{\lim ^{m}}^{m} \rightarrow \operatorname{Tel}^{\infty}(A .)
$$

Lemma 3.1. Let $L . K$. be a map of inverse systems of (bi-cosimplicial) CDGA's which is a quasi-isomorphism at each level. That is, each $L_{n} \rightarrow K_{n}$ is a quasiisomorphism. Suppose, furthermore, that the transition maps for both systems (denoted $\pi$ ) have the following property: there exists some $m$ such that for each element $x$ of level $i$, there exists an element $y$ of level $i+1$ such that $\pi(y)=p^{m} x$. Then

is a quasi-isomorphim.

$$
\underline{\lim }_{n} \lim ^{n} L . \underline{\lim }_{n} \varliminf_{\longleftarrow}{ }^{n} K
$$

Proof. It is straightforward to check that the twisted inverse limit commutes with the cone construction, so that, for each $n$, we have a distinguished triangle

$$
\varliminf^{n} L . \longrightarrow \varliminf^{n} K . \longrightarrow \varliminf^{n} C . \rightarrow L .[1]
$$


where $C$. is the cone of $L \rightarrow K$. Since direct limits commute with cohomology, we need only show that

$$
\lim _{\longrightarrow} H\left(\lim ^{n} C .\right)=0
$$

In fact, the transition map

$$
H\left(\lim ^{n} C .\right) \rightarrow H\left(\lim ^{n+m} C .\right)
$$

is zero. To see this, let the cocycle $\left(c_{i}\right)$ represent an element of $H\left(\lim ^{n} C\right.$.). We will show that $\left(p^{m i} c_{i}\right) \in \varliminf^{\lim }{ }^{n+m} C$. is a coboundary. Construct an element $\left(b_{i}\right) \in \varliminf^{n+m} C$. such that $d\left(b_{i}\right)=\left(p^{m i} c_{i}\right)$ inductively as follows: assume we have constructed up to $b_{j}$. That is, for $i \leq j, d b_{i}=p^{m i} c_{i}$ and $\pi\left(b_{j}\right)=p^{n+m} b_{j-1}$. Since each $C_{i}$ is acyclic, there exists an $x_{j+1} \in C_{j+1}$ such that $d x_{j+1}=c_{j+1}$. Hence, $d \pi\left(p^{m j} x_{j+1}\right)=p^{m j} \pi\left(c_{j+1}\right)=p^{m j+n} c_{j}=p^{n} d b_{j}$ and $d\left(\pi\left(p^{m j} x_{j+1}\right)-\right.$ $\left.p^{n} b_{j}\right)=0$. Again by acyclicity in level $j$, we can then find an $a_{j}$ such that $d a_{j}=\pi\left(p^{m j} x_{j+1}\right)-p^{n} b_{j}$. Multiplying by $p^{m}$, we get

$$
\pi\left(p^{(j+1) m} x_{j+1}\right)-p^{n+m} b_{j}=d\left(p^{m} a_{j}\right)=d \pi\left(a_{j+1}\right)
$$

for some $a_{j+1}$. Now put

$$
b_{j+1}:=p^{(j+1) m} x_{j+1}-d a_{j+1}
$$

Then $\pi\left(b_{j+1}\right)=p^{n+m} b_{j}$ and $d b_{j+1}=p^{(j+1) m} c_{j+1}$, so we are done.

We emphasize that $T e l^{\infty}$ is a functor from inverse systems of (bi-cosimplicial) CDGA's to (bi-cosimplicial) CDGA's and that the maps

$$
\varliminf_{\varlimsup} A . \rightarrow \varliminf_{m} \varliminf^{m} A . \rightarrow \operatorname{Tel}^{\infty}(A .)
$$

are multiplicative.

\section{Proof of theorem}

In this section, it will be useful to employ the following notation: Given a $p$-adic formal scheme $S$ and an integer $m, S / m$ denotes $S \otimes_{\mathbf{Z}} \mathbf{Z} / m$ which of course only depends on the p-adic valuation of $m$.

We denote by $S_{1}$ and $S_{2}$ the scheme Spec $W[t]$ equipped with the log structures associated to the pre-log structures $\mathbf{N} \rightarrow W[t]$ that send 1 to 0 and $t$, respectively. So both restrict to the $\log$ structure of the punctured point on $\operatorname{Spec}(k) . W[t]$ carries the lifting of Frobenius $\sigma$ given by the usual Frobenius on $W$ and sending $t$ to $t^{p}$. Denote by $W<<t>>$, the $p$-adic completion of the divided power polynomial algebra $W<t>$ over $W$ which therefore also carries log structures induced by those of $S_{1}$ and $S_{2}$. Denote these formal log schemes by $\hat{S}_{1}$ and $\hat{S}_{2}$. The Frobenius $\sigma$ of $W[t]$ naturally extends to $W<<t>>$. We will denote by $S_{i}^{(n)}$ the scheme $\operatorname{Spec}(W[t])$ equipped with the log structure of $S_{i}$ pulled back by the $n$-th iterate of the Frobenius. Hence, we see that $S_{1}^{(n)} \simeq S_{1}$ while the log structure on $S_{2}^{(n)}$ is associated to the pre-log structure $\mathbf{N} \rightarrow W[t]$ that sends 1 to $t^{p^{n}}$. We will also use the notation $\hat{S}_{i}^{(n)}$ in the obvious manner. 
The difficult part of the Hyodo-Kato isomorphism says that if $Y$ is a smooth fine proper log scheme of Cartier type, then

$$
H^{i}\left(Y / S_{1}\right) \otimes_{\mathbf{Z}} \mathbf{Q} \simeq H^{i}\left(Y / S_{2}\right) \otimes_{\mathbf{Z}} \mathbf{Q}
$$

that is, the crystalline cohomology is the same for the two log structures, up to isogeny.

We recall the proof, taking care to make some maps more explicit.

We start out by choosing embedding systems $Y \hookrightarrow Z^{1}$ and $Y \hookrightarrow Z^{2}$ for $Y$ with respect to the two $\log$ schemes $S_{1}$ and $S_{2}$ that admit Frobenius lifts. This notion requires a brief explanation: Denote by $S_{a}^{(n)}$ the Frobenius twisted log schemes introduced above. There are then maps $S_{a} \rightarrow S_{a}^{(n)}$ induced from the monoid map $\mathbf{N} \rightarrow \mathbf{N}, 1 \mapsto p^{n}$. Now, $\left(Z^{a}\right)^{(n)}$ is a smooth simplicial log scheme over $S_{a}^{(n)}$, and a Frobenius lift refers to a map $F: Z^{a} \rightarrow\left(Z^{a}\right)^{(1)}$ that fits into the commutative diagram

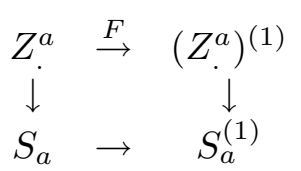

Such embedding systems can be constructed, for example, as follows: $Y_{0}$ in both cases is just the disjoint union of the elements of an affine open covering of $Y$ and $Z_{0}^{1}$ and $Z_{0}^{2}$ are smooth liftings of $Y_{0}$ to $S_{1}$ and $S_{2}$, respectively. Then the $Y_{i}=Y_{1} \times_{Y} Y_{1} \times \cdots \times Y_{1}(i+1$-times $)$ embed diagonally into

$$
Z_{i}^{a}=Z_{0}^{a} \times_{S_{a}} Z_{0}^{a} \times_{S_{a}} \cdots \times_{S_{a}} Z_{0}^{a}
$$

( $i+1$-times $)$ for $a=1,2$ and come together to form a simplicial hypercovering. By the affine smoothness, we easily get the Frobenius lifts in cosimplicial level 0 and then the other levels by taking products.

Let $C_{1}$ and $C_{2}$ be crystalline complexes for the two different embedding systems, and form the bi-cosimplicial algebras over $W<<t>>$

$$
B_{a}:=\lim _{\longleftarrow} \Gamma\left(G\left(C_{a}\right)\right)
$$

for $a=1,2$. Denote by $B_{a}^{(n)}$ the pull-back of $B_{a}$ via $\sigma^{n}$, which therefore arise from crystalline complexes for $\left(Z^{a}\right)^{(n)}$, the $n$-th Frobenius pull-back of $Z^{a}$, with respect to $S_{a}^{(n)}$. In particular, when we adjoin divided powers and reduce mod $p^{n}$ !, the two log structures are isomorphic, so we get isomorphisms in the derived category of bi-cosimplicial complexes

$$
B_{1}^{(n)} /\left(p^{n}\right) ! \simeq B_{2}^{(n)} /\left(p^{n}\right) !
$$

We can get these maps to fit into commutative diagrams of bi-cosimplicial CDGA's

$$
\begin{array}{ccccc}
B_{1}^{(n+1)} /\left(p^{n}\right) ! & \rightarrow & L_{n+1} & \leftarrow & B_{2}^{(n+1)} /\left(p^{n}\right) ! \\
\downarrow \phi & & \downarrow & & \downarrow \downarrow \\
B_{1}^{(n)} /\left(p^{n}\right) ! & \rightarrow & L_{n} & \leftarrow & B_{2}^{(n)} /\left(p^{n}\right) !
\end{array}
$$


where the horizontal arrows are multiplicative quasi-isomorphisms of CDGA's (as opposed to maps in the derived category of complexes) constructed as follows. The vertical arrows on either end are induced by the Frobenius lifts. $L_{n}$ is the crystalline complex for $Y^{(n)}$ relative to $\hat{S}_{1}^{(n)} / p^{n} !=\hat{S}_{2}^{(n)} / p^{n}$ ! computed using the 'diagonal' embedding system

$$
Y_{.}^{(n) \hookrightarrow}\left(Z_{.}^{1}\right)^{(n)} \times_{\hat{S}_{1}^{(n)} / p^{n !}}\left(Z_{.}^{2}\right)^{(n)}
$$

The Frobenius lifts for $Z^{1}$ and $Z^{2}$ determine one for each product giving the maps $L_{n+1} \rightarrow L_{n}$. The horizonal maps are then induced by the projections. We stress that all the maps are given by pull-backs of differential forms, and hence, are multiplicative.

On the other hand, the iterates of the Frobenius induce multiplicative maps of inverse systems

$$
B_{a}^{(.)} / p \cdot \rightarrow B_{a} / p \cdot
$$

where the target is just the inverse system given by the reductions of $B_{a}$ with the natural projections connecting them.

Lemma 3.1 admits the following corollaries:

Corollary 4.1. The following arrows are quasi-isomorphisms:

$$
\operatorname{Tel}^{\infty}\left(B_{1}^{(.)}\right) \rightarrow \operatorname{Tel}^{\infty}(L .) \leftarrow \operatorname{Tel}^{\infty}\left(B_{2}^{(.)}\right)
$$

\section{Corollary 4.2.}

$$
\underline{\lim }_{m} \varliminf^{\lim ^{m}\left(B_{a} / p !\right) \rightarrow T e l^{\infty}\left(B_{a} / p !\right)}
$$

is a quasi-isomorphism.

On the other hand, we have the

Proposition 4.3. The map

$$
B_{a}^{(.)} / p \cdot \rightarrow B_{a} / p !
$$

induces a quasi-isomorphism

$$
\operatorname{Tel}^{\infty}\left(B_{a}^{(\cdot)} / p^{\cdot} !\right) \simeq \operatorname{Tel}^{\infty}\left(B_{a} / p^{\cdot} !\right)
$$

for $a=1,2$.

Proof. We will omit the subscript $a$ from the notation. Hence, $S$ also refers to either $S_{1}$ or $S_{2}$.

If $C_{n}$ is the cone of $B^{(n)} / p^{n} ! \rightarrow B / p^{n}$ ! then $\operatorname{Tel}_{n}(C$.) is the cone of

$$
\operatorname{Tel}_{n}\left(B^{(\cdot)} / p \cdot\right) \rightarrow \operatorname{Tel}_{n}(B / p \cdot)
$$

That is, we have a distinguished triangle:

$$
0 \rightarrow \operatorname{Tel}_{n}\left(B^{(\cdot)} / p^{\cdot}\right) \rightarrow \operatorname{Tel}_{n}\left(B / p^{\cdot} !\right) \rightarrow \operatorname{Tel}_{n}(C .) \rightarrow \operatorname{Tel}_{n}\left(B^{(\cdot)} / p^{\cdot}\right)[1]
$$

The key point is the following

Lemma 4.4. $H^{i}\left(C_{n}\right)=H^{i}\left(\operatorname{Tel}_{n}(C).\right)$ is killed by $p^{2 i n}$ for $i>0$ and $p^{n}$ for $i=0$. 
Proof of lemma. This is essentially [6] (2.25) which is a logarithmic version of [2], chapter 8. However, we give here a self-contained proof for the convenience of the reader.

Since the algebras are constructed out of the stalks of Godements resolutions, the statement is local on $Y$. So we may assume that the embedding system is just a smooth $S$ lift $X$ and that we also have a Frobenius lift $f$. Thus, $f$ induces pull-back maps

$$
\phi^{n}: \Omega_{X}^{(n)} / S^{(n)} \rightarrow \Omega_{X / S}
$$

It suffices to show that the cone $C_{\text {Cne }}$ of this map mod $p^{n}$ ! has $i$-th cohomology killed by $p^{2 i n}$ for $i>0$ and $p^{n}$ for $i=0$. We just give the argument for $i>0$ since the $i=0$ case is an obvious modification.

Recall the map $F$ given by $\phi / p^{i}$ in degree $i$. The definition works just as in the usual case ([7] 0.2.3.3) using the $W$-flatness of the sheaf of differentials. $F$ induces an injection ([8] III.1.5.4):

$$
F: \Omega_{X^{(1)} / S^{(1)}}^{i} /\left[p^{n}\left(\Omega_{X^{(1)} / S^{(1)}}^{i}\right)+p d\left(\Omega_{X^{(1)} / S^{(1)}}^{i-1}\right)\right] \hookrightarrow \Omega_{X / S}^{i} /\left[p^{n}\left(\Omega_{X / S}^{i}\right)+d\left(\Omega_{X / S}^{i-1}\right)\right]
$$

This is an immediate consequence of the Cartier isomorphism, again as in the classical case.

Note the following corollary: given $x \in \Omega_{X^{(1)} / S^{(1)}}^{i}$, if $F(x)=p^{m} y$ for some $y$, then $x=p^{m} z$ for some $z$. This is obvious for $m=1$ from the above injection. Assume it for $m-1$. $F(x)=p^{m} y$ in any case implies $x=p^{m} w+p d u$. But then $F(p d u)=F(x)-F\left(p^{m} w\right)=p^{m}(y-F(w))$ so $F(d u)$ is divisible by $p^{m-1}$. Therefore, $d u=p^{m-1} v$ and $x$ is divisible by $p^{m}$.

By iterating the argument, we also see that if $x \in \Omega_{X^{(n)} / S^{(n)}}^{i}$ and $F^{n}(x)$ is divisible by $p^{m}$, then so is $x$.

Now, let

represent a cocycle in

$$
(a, b) \in \Omega_{X / S}^{i} \oplus \Omega_{X^{(n)} / S^{(n)}}^{i+1}
$$

$$
\left(\text { Cone }_{n}\right)^{i}=\left(\Omega_{X / S}^{i} \oplus \Omega_{X^{(n)} / S^{(n)}}^{i+1}\right) \otimes \mathbf{Z} / p^{n} !
$$

Then $\phi^{n}(b)-d a=p^{n} ! x$ and $d b=p^{n} ! y$. Write the first equality as

$$
F^{n}\left(p^{i n} b\right)=p^{n} ! x+d a
$$

and apply the injection above to get

$$
p^{i n} b=p^{n} ! w+p d s=p^{n} ! w+d c
$$

for some $w$ and $c$. We also get

$$
d\left(\phi^{n}(c)-p^{i n} a\right)=p^{i n} \phi^{n}(b)-p^{n} ! p^{i n} F^{n}(w)-p^{i n} d a=p^{n} ! p^{i n}\left(x-F^{n}(w)\right)
$$

That is,

Recall the formula

$$
\phi^{n}(c)-p^{i n} a \in d^{-1}\left(p^{n} ! p^{i n} \Omega_{X / S}^{i+1}\right)
$$

$$
d^{-1}\left(p^{n} \Omega_{X / S}^{i+1}\right)=\Sigma_{0 \leq k \leq n} p^{k} F^{n-k}\left(\Omega_{X^{(n-k)} / S^{(n-k)}}^{i}\right)+\sum_{0 \leq k \leq n-1} F^{k}\left(d \Omega_{X(k) / S^{(k)}}^{i-1}\right)
$$


whose proof also follows [7] 0.2.3.13 verbatim, as pointed out by Jannsen in the appendix of [5]. Thus, we have

$$
\begin{gathered}
\phi^{n}(c)-p^{i n} a=p^{n} ! p^{i n} z_{0}+p^{n} ! p^{-1} p^{i n} F\left(z_{1}\right)+\cdots+p^{n} ! p^{-n} p^{i n} F^{n}\left(z_{n}\right)+\cdots \\
F^{N}\left(z_{N}\right)+d u_{0}+F\left(d u_{1}\right)+\cdots+F^{N-1}\left(d u_{N-1}\right)
\end{gathered}
$$

where $N=v_{p}\left(p^{n} !\right)+i n$. We examine the terms in this equality after multiplying by another $p^{i n}$. We get that $\phi^{n}\left(p^{i n} c\right)-p^{2 i n} a$ is of the form $p^{n} ! p^{i n} z+\phi^{n}(l)+d u$. If we apply the differential $d$, we get that $d \phi^{n}(l)=\phi^{n}(d l)$ is divisible by $p^{i n} p^{n}$ !. Thus, $F^{n}(d l)$ is divisible by $p^{n}$ !, and hence, so is $d l$. So we get $d\left(p^{i n} c-l\right)=$ $p^{2 i n} b\left(\bmod p^{n} !\right)$ and $\phi^{n}\left(p^{i n} c-l\right)-d u=p^{2 i n} a\left(\bmod p^{n} !\right)$. Hence, the class of $\left(p^{2 i n} a, p^{2 i n} b\right)\left(\bmod p^{n} !\right)$ is a coboundary. This proves the lemma.

For all our considerations, we may assume that the dimension $d$ of $Y$ is positive. Therefore, we see that all the cohomology of $C_{n}$ is killed by $p^{2 d n}$. The remainder of the proof of the proposition is as in the proof of Lemma 3.1: Let $\left(c_{n}\right)$ be a cocycle in $T e l^{m}(C$.$) . Then \left(p^{(2 d+1) n} c_{n}\right) \in T e l^{(m+2 d+1)}(C$. $)$ is a coboundary.

Remark 4.5. The fact that the relative Frobenius is an isogeny has been the source of many important theorems on crystalline cohomology starting with the theorem of Berthelot and Ogus. Here, it is rather mysteriously manifested in the isomorphism of infinitely twisted mapping telescopes.

Thus we have arrived at an explicit isomorphism in the derived category from

$$
\varliminf^{m} \varliminf^{m} B_{1} / p \text { ! }
$$

to

$$
\underline{\lim }^{m} \varliminf^{m} B_{2} / p \cdot
$$

mediated by the following system of arrows all of which are quasi-isomorphisms:

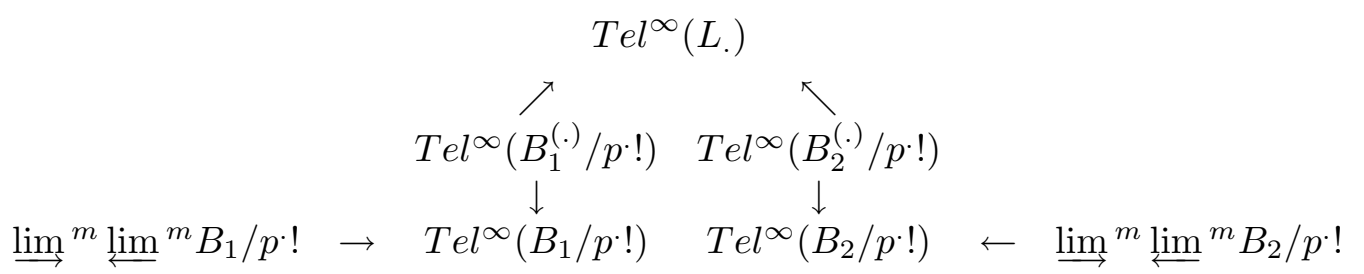

On the other hand, as has already been pointed out, it is trivial to check that if $L$. is an inverse system of $W$-algebras, then $\underline{\lim }^{m} \varliminf^{m} L$. is a $W$ algebra, even though each $\varliminf^{m} L$. separately is not. Also, maps in the category of inverse systems of algebras induce algebra homomorphisms for this double limit. Therefore, we see that the isomorphism constructed above is actually an isomorphism in the homotopy category of algebras.

Now consider the natural map

$$
\varliminf B_{a} / p ! \rightarrow \underline{\lim }^{m} \varliminf^{m} B_{a} / p \cdot
$$

By Ogus ([12] Lemma 18), we have an isomorphism

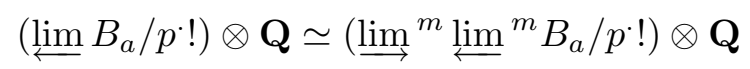


Thus, we have constructed a quasi-equivalence from

$$
\left(\lim _{\longleftarrow} B_{1} / p^{i} !\right) \otimes \mathbf{Q}
$$

to

$$
\left(\lim _{\longleftarrow} B_{2} / p^{i} !\right) \otimes \mathbf{Q}
$$

Applying the Thom-Whitney simple object functor twice, we get a quasiequivalence

$$
T W\left(C\left(Y, Z_{.}^{1}\right) \simeq T W\left(C\left(Y, Z_{.}^{2}\right)\right)\right.
$$

It is straightforward to check that this map is independent (in the homotopy category) of the various choices made.

The rest of the argument is as in [9], section 7, and amounts to a 'BerthelotOgus' type argument [2]. Choose a uniformizer $\pi$ of $A$ which therefore determines a presentation $A \simeq W[t] /(f(t))$, where $f(t)$ is an Eisentein polynomial of degree $e=[F: K]$. Let $R$ be the $p$-adic completion of the divided power envelop of $(f(t), p)$ inside $W[t]$. Thus $R$ is also the completed DP envelop of the ideal $\left(t^{e}\right)$. We have a natural map $g: R \rightarrow W<<t>>$. On the other hand, if $r$ is such that $p^{r} \geq e$, then the map $\sigma^{r}: W<<t>>\rightarrow W<<t>>$ factors through $W<<t>>\rightarrow R \rightarrow W<<t>>$. Composing in the other direction $R \rightarrow W<<$ $t>>\rightarrow R$ is, by definition, the Frobenius map of $R$. Let $Y^{\prime}=X \otimes A / p$ with the induced $\log$ structure. Also, give $\operatorname{Spec}(R)$ the $\log$ structure induces from $S_{2}$. We have the commutative diagram

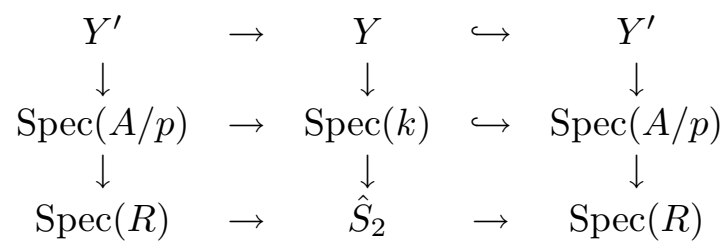

where the composite of the horizontal arrows are all the $r$-th iterate of the Frobenius. We choose crystalline complexes for $Y$ and $Y^{\prime}$ as follows. Construct first embedding systems for $Y$ and $Y^{\prime}$ with respect to $S_{2}$ that fit into a diagram

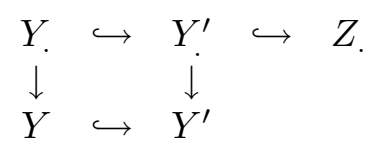

where $Z$. is smooth over $S_{2}$ and the left hand square is cartesian. We can also arrange for $Z$. to admit a Frobenius lift compatible with the Frobenius of $W[t]$. Then $C=\Omega_{Z . / W[t]} \otimes W<<t>>$ and $C^{\prime}=\Omega_{Z . / W[t]} \otimes R$ are crystalline complexes for $Y$ and $Y^{\prime}$ and we can regard both as simplicial sheaves of CDGA's on $Y$.

Denote by $C^{(r)}$ (resp. $\left(C^{\prime}\right)^{(r)}$ ) the pull-back of $C$ (resp. $C^{\prime}$ ) by the $r$-th power of the Frobenius map of $W<<t>>$ (resp. $R$ ). Then the big diagram above implies that

$$
\left(C^{\prime}\right)^{(r)} \simeq C^{(r)} \otimes_{W<<t>>} R
$$


in the homotopy category of sheaves of CDGA's on $Y_{\text {. }}$. On the other hand, the Frobenius lifts induce maps $C^{(r)} \rightarrow C$ and $\left(C^{\prime}\right)^{(r)} \rightarrow C^{\prime}$. So we have maps of sheaves of CDGA's

$$
C \otimes R \leftarrow C^{(r)} \otimes R \simeq\left(C^{\prime}\right)^{(r)} \rightarrow C^{\prime}
$$

and taking Thom-Whitney algebras, we have multiplicative maps

$$
T W(C) \otimes(R \otimes \mathbf{Q}) \leftarrow T W\left(C^{(r)}\right) \otimes(R \otimes \mathbf{Q}) \simeq T W\left(\left(C^{\prime}\right)^{(r)}\right) \rightarrow T W\left(C^{\prime}\right)
$$

of CDGA's over $R \otimes \mathbf{Q}$. From the fact that the relative Frobenius is an isogeny ([6] Proposition 2.24), we know that all these maps are quasi-equivalences. Now we tensor with the quotient map $R \otimes \mathbf{Q} \rightarrow A \otimes \mathbf{Q}=F$ to get

$$
T W(C) \otimes_{W<<t>>\otimes \mathbf{Q}} F \simeq T W\left(C^{\prime}\right) \otimes_{R \otimes Q} F
$$

By using the fact that $C^{\prime} \otimes A$ is the crystalline complex associated to an embedding system for $Y^{\prime}$ w.r.t. $A$ which is also true of $\Omega_{\hat{X} / A}$, we get

$$
T W\left(C^{\prime}\right) \otimes F \simeq T W\left(\Omega_{\hat{X} / A}\right) \simeq T W\left(\Omega_{X_{F} / F}\right)
$$

On the other hand, $C$ is quasi-equivalent to $C\left(Y ., Z^{2}\right)$ from the previous section, so that $T W(C) \simeq T W\left(C\left(Y ., Z^{2}\right)\right) \simeq T W\left(C\left(Y ., Z^{1}\right)\right)$ and $C\left(Y ., Z^{1}\right)$ is quasiequivalent to the base change to $W<<t>>$ of a crystalline complex for $Y$ with respect to $W$ so we get

$$
T W(C) \otimes F \simeq T W\left(W \omega_{Y}\right) \otimes_{K} F
$$

giving us the desired quasi-equivalence

$$
T W\left(W \omega_{Y}\right) \otimes_{K} F \simeq T W\left(\Omega_{X_{F} / F}\right)
$$

This is the isomorphism of homotopy types stated in the theorem.

Proof of corollary 1.2

We need to discuss basepoints. We start with a careful discussion of the basepoint for $W \omega_{Y}$. If $y: \operatorname{Spec}(k) \rightarrow Y$ is a point of $Y$, then there is a map $W \omega_{Y} \rightarrow y_{*}(W)$. It is given by 0 in positive degrees and the canonical map $e_{y}$ : $W \mathcal{O}_{Y} \rightarrow W(k)$ induced by $\mathcal{O}_{Y} \rightarrow k$. This map induces $T W\left(W \omega_{Y}\right) \rightarrow T W\left(y_{*}(W)\right)$. However, a simple examination of the definition yields the following description of the degree zero term $T W^{0}\left(y_{*}(W)\right)$ : It consists of collections $\left(f_{n}\right)$ where $f_{n}$ is a function on $\mathbf{A}_{K}^{n}$ with the property that $\partial_{i}\left(f_{n+1}\right)=f_{n}$ for any $i$. The map $\left(f_{n}\right) \rightarrow f_{0}$ yields a quasi-isomorphism $T W\left(y_{*}(W)\right) \simeq K$. We obtain thereby the augmentation map $T W\left(W \omega_{Y}\right) \rightarrow K$. Let's describe this map explicitly. The degree zero term of $T W\left(W \omega_{Y}\right)$ consists of compatible sequences ([9] section 3) $\left(a_{n}\right), a_{n} \in \prod_{\bar{p}}\left(W \mathcal{O}_{Y}\right)_{\bar{p}} \otimes \mathcal{O}_{\mathbf{A}_{K}^{n}}$ where $\bar{p}$ is an $n+1$-tuple of points in $Y$. Thus, $a_{0}$ is just an element of $\prod_{p}\left(W \mathcal{O}_{Y}\right)_{p}$, and the map $T W\left(W \omega_{Y}\right) \rightarrow K$ is zero in positive degrees while in degree zero it sends $\left(a_{n}\right)$ to $e_{y}\left(a_{0}\right)$. Now, let $(Y, Z$. $)$ be an embedding system for which $Y_{0}$ is the disjoint union of an affine open cover of $Y$ and $Z_{0}$ is a smooth lifting of $Y_{0}$ which admits a Frobenius lift $F$. By the construction at the beginning of the section, for example, such embedding systems exist. The base point $y \in Y$ lifts to $Y_{0}$ and a $W$ point $z$ of 
$Z=Z_{0}$. Locally, we can express $W_{n} \mathcal{O}_{Y}$ as the cohomology $\underline{H}^{0}\left(\Omega_{Z_{n} / W_{n}}\right)$ and then we get the map $\mathcal{O}_{Z} \rightarrow W \mathcal{O}_{Y}$ that sends $a$ to the sequence $\left(F^{n}(a)\left(\bmod p^{n}\right)\right)_{n}$ which is the degree zero component of the quasi-isomorphism $\Omega_{Z / W} \rightarrow W \omega_{Y}$. Let $\Omega_{Z / W} \otimes D_{y}(Z)$ be the divided power envelop of $y$ in $Z$. Then we have $\left(\underline{H}^{0}\left(\Omega_{Z_{n} / W_{n}} \otimes D_{y}\left(Z_{n}\right)\right)\right)_{n} \simeq W(k)$. The augmentation $e_{z}: \mathcal{O}_{Z} \rightarrow W$ given by evaluation at $z$ then fits into a commutative diagram

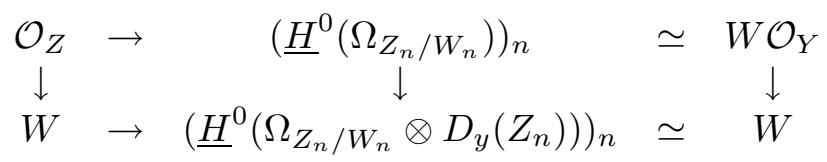

where the first arrow in the bottom row sends $a \in W$ to the inverse system $\left(\sigma^{n}(a)\left(\bmod p^{n}\right)\right)_{n}$ This implies that

$$
\left(T W\left(C\left(Y, Z_{.}\right)\right), e_{z}\right) \simeq\left(T W\left(W \omega_{Y}\right), e_{y}\right)
$$

as augmented algebras.

Now, assume we are in the situation at the end of section 2 where $V \rightarrow \operatorname{Spec}(R)$ is a smooth proper connected fine saturated log scheme over an affine base and $\operatorname{Spec}(R) \hookrightarrow \operatorname{Spec}(B)$ is an exact topologically nilpotent immersion where $B$ is of characteristic zero. Assume also that we are given a point $v: \operatorname{Spec}(R) \rightarrow V$. Then we can always find an embedding system $(V, Z$. $)$ with the property that $v$ lifts to $V_{0}$ and to a point $z: B \rightarrow Z_{0}$. This point allows us to put an augmentation $T W(C(V, Z).) \rightarrow B \otimes \mathbf{Q}$ on the Thom-Whitney algebra and a product construction shows that for any two choices of embedding systems, there is a homotopy equivalence between the Thom-Whitney algebras which is compatible with the augmentation. Similarly for different choices of liftings of the point $y$. From this, applied to the various embedding systems that occur in the proof of the theorem, one easily deduces that the homotopy equivalence

$$
T W\left(C\left(Y, Z_{.}\right)\right) \otimes F \simeq T W\left(\Omega_{X_{F}}\right)
$$

takes the augmentation induced by $e_{z}$ to that induced by evaluation at $x_{F}$.

Proof of Corollary 1.3.

The isomorphism classes of the higher rational homotopy groups are determined by their dimension, and this dimension can be computed in any complex embedding of $E$ or after base change to the completion $E_{v}$ of $E$ w.r.t. $v$. The assumptions imply that the special fibers $Y_{1}$ and $Y_{2}$ are isomorphic smooth log schemes. Thus, $T W\left(\omega_{Y_{2}}\right) \simeq T W\left(\omega_{Y_{2}}\right)$, which implies the quasi-equivalence of $T W\left(\Omega_{X_{E}(1)}\left(\log D_{E}(1)\right)\right) \otimes E_{v}$ and $T W\left(\Omega_{X_{E}(2)}\left(\log D_{E}(2)\right)\right) \otimes E_{v}$. Thus, their bar complexes are quasi-equivalent, giving isomorphisms of their cohomology groups, i.e., the higher De Rham homotopy groups of $X_{E}(1)-D_{E}(1)$ and $X_{E}(2)-D_{E}(2)$ [13]. 


\section{Acknowledgement}

Both authors were supported in part by grants from the National Science Foundation. We are also very grateful to the referee who read an earlier version very carefully and made many useful suggestions. He/she also corrected a defective proof involved in the definition of the rational homotopy type.

\section{References}

[1] M. Artin, B. Mazur, Etale homotopy. Reprint of the 1969 original. Lecture Notes in Mathematics, 100. Springer-Verlag, Berlin, 1986. iv+169 pp.

[2] P. Berthelot, A. Ogus, F-isocrystals and de Rham cohomology. I. Invent. Math. 72 (1983), 159-199.

[3] R. Godement, Topologie algébrique et théorie des faisceaux. (Actualités Sci. Ind. 1252. Publ. Math. Univ. Strasbourg. Hermann, Paris 1958

[4] A. Grothendieck, Éléments de géométrie algébrique. III. Étude cohomologique des faisceaux cohérents. II. Inst. Hautes Études Sci. Publ. Math. 17 (1963) 91 pp.

[5] O. Hyodo, On the de Rham-Witt complex attached to a semi-stable family. Compositio Math. 78 (1991), 241-260.

[6] O. Hyodo, K. Kato, Semi-stable reduction and crystalline cohomology with logarithmic poles. Périodes p-adiques (Bures-sur-Yvette, 1988). Astérisque 223 (1994), 221-268.

[7] L. Illusie, Complexe de de Rham-Witt et cohomologie cristalline. (French) Ann. Sci. cole Norm. Sup. 12 (1979), 501-661.

[8] L. Illusie, M. Raynaud, Les suites spectrales associées au complex de De Rham-Witt. Publ. Math. IHES 57 (1983), 73-212.

[9] M. Kim, R. M. Hain, A De Rham-Witt approach to crystalline rational homotopy theory. (Preprint 2002). Available as math.AG/0105008.

[10] J. W. Morgan, The rational homotopy theory of smooth, complex projective varieties (following P. Deligne, P. Griffiths, J. Morgan, and D. Sullivan) (Invent. Math. 29 (1975), 245-274). Séminaire Bourbaki, Vol. 1975/76, 28ème année, Exp. No. 475, pp. 69-80. Lecture Notes in Math., Vol. 567, Springer, Berlin, 1977.

[11] V. Navarro-Aznar, Sur la théorie de Hodge-Deligne. Invent. Math. 90 (1987), 11-76.

[12] A. Ogus, F-crystals on schemes with constant log structure. Special issue in honour of Frans Oort. Compositio Math. 97 (1995), 187-225.

[13] Z. Wojtkowiak, Cosimplicial objects in algebraic geometry. Algebraic $K$-theory and algebraic topology (Lake Louise, AB, 1991), 287-327, NATO Adv. Sci. Inst. Ser. C Math. Phys. Sci., 407, Kluwer Acad. Publ., Dordrecht, 1993.

Department of Mathematics, University of Arizona, Tucson, AZ, 85721

E-mail address: kim@math.arizona.edu

Department of Mathematics, Duke University, Durham, NC, 27708

E-mail address: hain@math.duke.edu 